\title{
Stability of optical caustics with $r$-corners
}

\author{
Takaharu TSUKADA
}

(Received August 25, 1997)

\begin{abstract}
In this papar, we investigate the stability of the optical caustic generated by a light source hypersurface with an $r$-corner in a smooth manifold under a fixed Hamiltonian system. Main results are the stability of optical caustics under the perturbation of hypersurfaces and a realization of a caustic as a stable optical caustic generated by some hypersurfaces.
\end{abstract}

Key words: lagrangian singularity, caustic, singularity.

\section{Introduction}

In [5] K. Jänich explained the wavefront propagation mechanism on a manifold which is completely described by a positive and positively homogeneous Hamiltonian function on the cotangent bundle and investigated the local gradient models given by the ray length function. He considered the case when the initial wave front is a smooth hypersurface without boundary. This case is corresponding to the theory of Lagrangian singularities (cf., [1]).

In this paper we consider the case when the initial wave front is a hypersurface with an $r$-corner $(\S 1)$. The rays incident to conormal directions from each edges of the hypersurface gives a regular $r$-cubic configuration (cf., Section 3) at a point in the cotangent bundle, which is a generalized notion of Lagrangian submanifolds. The optical caustic with an r-corner generated by the hypersurface is given as the caustic of the regular $r$-cubic configuration. The notion of regular $r$-cubic configurations in complex analytic category has been introduced in [3], [4] and the real version has been developed in [8]. In [8] we have shown that any regular $r$-cubic configuration (at least locally) has a generating family which is a kind of families of functions. We also have shown that the stability of regular $r$-cubic configuration corresponds to the stable generating family.

In this paper we consider the following problems, extending of the investigations by K. Jänich [5] and G. Wassermann [10]: For a fixed Hamiltonian 
function on the cotangent bundle;

(1) Is the stability of the optical caustic with the $r$-corner under perturbations of the hypersurface equivalent to the stability of a generating family of the corresponding regular $r$-cubic configuration?

(2) For a given function germs, when does there exist a light source hypersurface germ with an $r$-corner which satisfy the following conditions (a) (b)?: a) A generating family of the corresponding regular $r$-cubic configuration is an unfolding of the given function germ, b) The optical caustic with the $r$-corner generated by the hypersurface is stable.

The answer of (1) is 'Yes'. This means that the classification stable optical caustics with $r$-corners is reduced to the classification of stable generating family under the reticular $R^{+}$-equivalence (cf., Section 3 ).

We give a partial answer to the probrem (2). The answer of (2) gives us a method to decide when the caustic defined by a function germ in the classification list can be realized as a stable optical caustic with an $r$-corner for a fixed Hamiltonian function.

In the investigations by K. Jänich and G. Wassermann the R-L-equivalence was used as the equivalence relation of function germs. Instead, we use the reticular $\mathrm{R}^{+}$-equivalence as the equivalence relations because the $\mathrm{R}^{+}$-equivalence among generating families is naturally used in the theory of Lagrangian singularities (cf., [1]) and the reticular $\mathrm{R}^{+}$-equivalence relation is its extension.

In $\S 1$, we introduce the basic notations and setting of this paper. In $\S 2$, we give a brief summary of the theory of regular $r$-cubic configurations. In $\S 3$, we recall the deformation theory of function germs with respect to the reticular $\mathrm{R}^{+}$-equivalence. The main Theorem 5.2, which solves the first probrem in Introduction, is formulated and proved in $\S 4$. In the last section we solve the second probrem under some conditions on the Hamiltonian function.

\section{Preliminaries}

Fix non-negative integers $r$ and $k$. Let $M$ be an $m(=r+k+1)$ dimensional differentiable manifold and $H: T^{*} M \backslash 0 \rightarrow \mathbf{R}$ be a $C^{\infty}$-function, called a Hamiltonian function. We suppose that $H$ is everywhere positive and positively homogeneous of degree one, that is $H(\lambda \xi)=\lambda H(\xi)$ for all $\lambda>$ 0 and $\xi \in T^{*} M \backslash 0$. Let $X_{H}$ denote the corresponding Hamiltonian vector 
field on $T^{*} M \backslash 0$. Then $X_{H}$ is given locally by the Hamiltonian equations:

$$
\dot{q}_{i}=\frac{\partial H}{\partial p_{i}}, \quad \dot{p}_{i}=-\frac{\partial H}{\partial q_{i}},
$$

where $(q, p)$ are local canonical coordinates of $T^{*} M$.

We set $E=H^{-1}(1)$ and consider the following canonical projections $\pi_{M}: T^{*} M \rightarrow M, \pi_{E}: \mathbf{R} \times E \rightarrow E, \pi_{\mathbf{R}}: \mathbf{R} \times E \rightarrow \mathbf{R}$. We denote by $E_{q}$ the fiber of the spherical cotangent bundle $\left.\pi\right|_{E}$ at $q \in M$.

Let $q_{0} \in M, t_{0} \geq 0, \xi_{0} \in E_{q_{0}}$ and $\eta_{0}$ the image of the phase flow of $X_{H}$ at $\left(t_{0}, \xi_{0}\right)$. Since the phase flow of $X_{H}$ preserves values of $H$, the local phase flow $\Psi:\left(\mathbf{R} \times T^{*} M \backslash 0,\left(t_{0}, \xi_{0}\right)\right) \rightarrow\left(T^{*} M \backslash 0, \eta_{0}\right)$ of $X_{H}$ induces the map $\Phi:\left(\mathbf{R} \times E,\left(t_{0}, \xi_{0}\right)\right) \longrightarrow\left(\mathbf{R} \times E,\left(t_{0}, \eta_{0}\right)\right)$ given by $\Phi(t, \xi)=(t, \Psi(t, \xi))$.

We set $\exp =\pi_{M} \circ \Phi:\left(\mathbf{R} \times E,\left(t_{0}, \xi_{0}\right)\right) \rightarrow\left(M, u_{0}\right), \exp _{q_{0}}=\left.\exp \right|_{\mathbf{R} \times E_{q_{0}}}$, $\exp ^{-}=\pi_{M} \circ \Phi^{-1}:\left(\mathbf{R} \times E,\left(t_{0}, \eta_{0}\right)\right) \rightarrow\left(M, q_{0}\right), \exp _{u_{0}}^{-}=\left.\exp ^{-}\right|_{\mathbf{R} \times E_{u_{0}}}, \phi_{1}=$ $\left(\pi_{M}, \exp \right):\left(\mathbf{R} \times E,\left(t_{0}, \xi_{0}\right)\right) \rightarrow\left(M \times M,\left(q_{0}, u_{0}\right)\right), \phi_{2}=\left(\exp ^{-}, \pi_{M}\right):(\mathbf{R} \times$ $\left.E,\left(t_{0}, \eta_{0}\right)\right) \rightarrow\left(M \times M,\left(q_{0}, u_{0}\right)\right)$, where $u_{0}=\pi_{M}\left(\eta_{0}\right)$. Then the following diagram is commutative:

$$
\begin{aligned}
& \left(\mathbf{R} \times E,\left(t_{0}, \xi_{0}\right)\right) \quad \stackrel{\Phi}{\longrightarrow} \quad\left(\mathbf{R} \times E,\left(t_{0}, \eta_{0}\right)\right) \\
& \swarrow \exp \quad \phi_{1} \searrow \swarrow \phi_{2} \quad \exp ^{-} \searrow \\
& \left(M, u_{0}\right) \quad \stackrel{\pi_{2}}{\longleftarrow} \quad\left(M \times M,\left(q_{0}, u_{0}\right)\right) \quad \stackrel{\pi_{1}}{\longrightarrow} \quad\left(M, q_{0}\right)
\end{aligned}
$$

By $[5,2.2]$ we have the following proposition

Proposition 2.1 If $\exp _{q_{0}}$ is regular then $\phi_{1}$ and $\phi_{2}$ are diffeomorphisms.

If $\exp _{q_{0}}$ is regular, then we define the function germ

$$
\tau=\pi_{\mathbf{R}} \circ \phi_{1}^{-1}=\pi_{\mathbf{R}} \circ \phi_{2}^{-1}:\left(M \times M,\left(q_{0}, u_{0}\right)\right) \rightarrow\left(\mathbf{R}, t_{0}\right) .
$$

We call $\tau$ the ray length function associated with the regular point $\left(t_{0}, \xi_{0}\right)$ of $\exp _{q_{0}}$. Set $\xi=\pi_{E} \circ \phi_{1}^{-1}:\left(M \times M,\left(q_{0}, u_{0}\right)\right) \rightarrow\left(E, \xi_{0}\right), \eta=$ $\pi_{E} \circ \phi_{2}^{-1}:\left(M \times M,\left(q_{0}, u_{0}\right)\right) \rightarrow\left(E, \eta_{0}\right)$. By [5, Lemma 2] we have

$$
\begin{aligned}
d_{q} \tau(q, u)=-\xi(q, u), d_{u} \tau(q, u)= & \eta(q, u) \\
& \text { for }(q, u) \in\left(M \times M,\left(q_{0}, u_{0}\right)\right)
\end{aligned}
$$

Let $\mathbf{H}^{r}=\left\{\left(x_{1}, \ldots, x_{r}\right) \in \mathbf{R}^{r} \mid x_{1} \geq 0, \ldots, x_{r} \geq 0\right\}$ be an $r$-corner. Let $V^{0}$ be the hypersurface germ in $\left(M, q_{0}\right)$ satisfying $\left.\xi_{0}\right|_{T_{q_{0}} V^{0}}=0$ with an $r$-corner defined as the image of an immersion $\iota:\left(\mathbf{H}^{r} \times \mathbf{R}^{k}, 0\right) \rightarrow\left(M, q_{0}\right)$. 
We parameterize $V^{0}$ by $\iota$.

From now on, we fix an $m(=r+k+1)$-dimensional manifold $M$, a Hamiltonian function $H: T^{*} M \backslash 0 \rightarrow \mathbf{R}, q_{0} \in M, \xi_{0} \in E_{q_{0}}$ and $t_{0} \geq 0$. We suppose that $\left(t_{0}, \xi_{0}\right)$ be a regular point of the ray length function $\tau$ of $\exp _{q_{0}}$ and put $\eta_{0}=\pi_{E} \circ \Phi\left(t_{0}, \xi_{0}\right), u_{0}=\pi_{M}\left(\eta_{0}\right)$.

\section{Regular $r$-cubic configuration associated with a light source hypersurface with an $r$-corner}

We now give a brief summary of the theory of regular $r$-cubic configurations which has been developed in [8].

Set $\Lambda_{\sigma}^{0}=\left\{(q, p) \in\left(T^{*} \mathbf{R}^{m}, 0\right) \mid q_{\sigma}=p_{I_{r}-\sigma}=q_{r+1}=\cdots=q_{m}=0\right.$, $\left.q_{I_{r}-\sigma} \geq 0\right\}$ for $\sigma \subset I_{r}=\{1, \ldots, r\}$, where $(q, p)$ are canonical coordinates of $\left(T^{*} \mathbf{R}^{m}, 0\right)$

Definition 3.1 Let $\eta \in T^{*} M \backslash 0$ and $\Lambda_{\sigma}$ be a lagrangian submanifold of $\left(T^{*} M \backslash 0, \eta\right)$ for $\sigma \subset I_{r}$. We call $\left\{\Lambda_{\sigma}\right\}_{\sigma \subset I_{r}}$ a regular $r$-cubic configuration if there exists a symplectomorphism germ $S:\left(T^{*} \mathbf{R}^{m}, 0\right) \rightarrow\left(T^{*} M \backslash 0, \eta\right)$ such that $\Lambda_{\sigma}=S\left(\Lambda_{\sigma}^{0}\right)$ for $\sigma \subset I_{r}$. The caustic of $\left\{\Lambda_{\sigma}\right\}_{\sigma \subset I_{r}}$ is defined by the union of the critical values of $\left.\pi\right|_{\Lambda_{\sigma}}$ for $\sigma \subset I_{r}$ and $\pi\left(\Lambda_{\sigma} \cap \Lambda_{\tau}\right)$ for $\sigma \neq \tau \subset I_{r}$.

Equivalence relations of regular $r$-cubic configurations: Let $\eta_{1}, \eta_{2} \in$

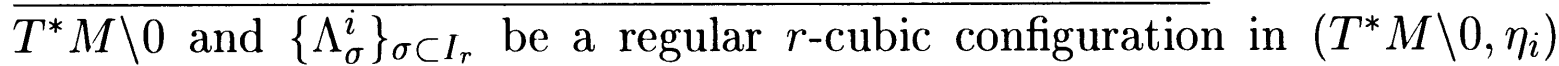
for $i=1,2$. We say that $\left\{\Lambda_{\sigma}^{1}\right\}_{\sigma \subset I_{r}}$ and $\left\{\Lambda_{\sigma}^{2}\right\}_{\sigma \subset I_{r}}$ are lagrangian equivalent if there exists a lagrangian equivalence $\Theta:\left(T^{*} M \backslash 0, \eta_{1}\right) \rightarrow\left(T^{*} M \backslash 0, \eta_{2}\right)$ such that $\Theta\left(\Lambda_{\sigma}^{1}\right)=\left(\Lambda_{\sigma}^{2}\right)$ for $\sigma \subset I_{r}$.

Generating families: Let $\mathcal{E}(r ; s)$ be the set of smooth function germs on $\overline{\left(\mathbf{H}^{r} \times \mathbf{R}^{s}, 0\right)}$ and let $\mathrm{m}(r ; s)=\{f \in \mathcal{E}(r ; s) \mid f(0)=0\}$ be its maximal ideal. Let $\eta \in T^{*} M \backslash 0$. We say that $F(x, y, u) \in \mathrm{m}(r ; s+m)$, where $x \in \mathbf{H}^{r}, y \in \mathbf{R}^{s}$ and $u \in \mathbf{R}^{m}$, is a generating family of a regular $r$-cubic configuration $\left\{\Lambda_{\sigma}\right\}_{\sigma \subset I_{r}}$ in $\left(T^{*} M \backslash 0, \eta\right)$ if $F$ is non-degenerate, that is

$\operatorname{rank}\left(\begin{array}{cc}\frac{\partial^{2} F}{\partial x \partial y} & \frac{\partial^{2} F}{\partial x \partial u} \\ \frac{\partial^{2} F}{\partial y \partial y} & \frac{\partial^{2} F}{\partial y \partial u}\end{array}\right)_{0}=r+s$

and $\left.F\right|_{x_{\sigma}=0}$ is generating family of $\Lambda_{\sigma}$ under an identification of $(M, \pi(\eta))$ 
and $\left(\mathbf{R}^{m}, 0\right)$, that is

$$
\begin{aligned}
\Lambda_{\sigma}=\left\{d_{u} F(x, y, u) \in\right. & \left(T^{*} M \backslash 0, \eta\right) \mid \\
& \left.x_{\sigma}=d_{x_{I_{r}-\sigma}} F(x, y, u)=d_{y} F(x, y, u)=0\right\}
\end{aligned}
$$

for suitable coordinates $\left(u_{1}, \ldots, u_{m}\right)$ of $(M, \pi(\eta))$.

Equivalence relations of generating families: We denote $\mathcal{B}(r ; l)$ the set of diffeomorphism germs on $\left(\mathbf{H}^{r} \times \mathbf{R}^{l}, 0\right)$ which preserve $\left(\mathbf{H}^{r} \cap\left\{x_{\sigma}=0\right\}\right) \times \mathbf{R}^{l}$ for all $\sigma \subset I_{r}$. We say that function germs $f, g \in \mathrm{m}(r ; s)$ are reticular $R$-equivalent if there exists $\phi \in \mathcal{B}(r ; s)$ such that $g=f \circ \phi$. We say that function germs $F(x, y, v), G(x, y, v) \in \mathrm{m}(r ; s+n)$, where $x \in \mathbf{H}^{r}, y \in \mathbf{R}^{s}$ and $v \in \mathbf{R}^{n}$, are reticular $R^{+}$-equivalent (as $n$-dimensional unfoldings) if there exist $\Phi \in \mathcal{B}(r ; s+n)$ and $\alpha \in \mathrm{m}(n)$ satisfying the following:

(1) $\Phi=(\phi, \psi)$, where $\phi:\left(\mathbf{H}^{r} \times \mathbf{R}^{s+n}, 0\right) \rightarrow\left(\mathbf{H}^{r} \times \mathbf{R}^{s}, 0\right)$ and $\psi:$ $\left(\mathbf{R}^{n}, 0\right) \rightarrow\left(\mathbf{R}^{n}, 0\right)$.

(2) $G(x, y, v)=F(\phi(x, y, v), \psi(v))+\alpha(v)$ for $(x, y, v) \in\left(\mathbf{H}^{r} \times \mathbf{R}^{s+n}, 0\right)$. We say that function germs $F\left(x, y_{1}, \ldots, y_{s_{1}}, v\right) \in \mathrm{m}\left(r ; s_{1}+n\right)$ and $G\left(x, y_{1}, \ldots, y_{s_{2}}, v\right) \in \mathrm{m}\left(r ; s_{2}+n\right)$ are stably reticular $R^{+}$-equivalent if $F$ and $G$ are reticular $\mathrm{R}^{+}$-equivalent after the addition of non-degenerate quadratic forms in the variables $y$.

Theorem 3.2 [8] Two regular $r$-cubic configurations defined at some points in $T^{*} M \backslash 0$ are lagrangian equivalent if and only if their generating families are stably reticular $R^{+}$-equivalent.

For each $\sigma \subset I_{r}$ we define $L_{\sigma}^{0}$ by the set of conormal vectors of $V_{\sigma}^{0}:=$ $V^{0} \cap\left\{x_{\sigma}=0\right\}$ in $\left(E, \xi_{0}\right)$ as the initial rays incident from $V_{\sigma}^{0}$. Then we regard the set $\Lambda_{\sigma}$ the image of covectors in $L_{\sigma}^{0}$ by $\pi_{E} \circ \Phi$ around time $t_{0}$, that is

$$
\Lambda_{\sigma}=\left\{\pi_{E} \circ \Phi(t, \xi) \in\left(E, \eta_{0}\right) \mid(t, \xi) \in\left(\mathbf{R}, t_{0}\right) \times L_{\sigma}^{0}\right\} .
$$

Then we regard $\Lambda_{\sigma}$ as the set of rays incident from $V_{\sigma}^{0}$ at time $t_{0}$. We also regard the union of $\Lambda_{\sigma}$ for all $\sigma \subset I_{r}$ as the set of rays incident from the hypersurface $V^{0}$ at time $t_{0}$.

We now prove that, if $\exp _{q_{0}}$ is regular, then a hypersurface germ in $\left(M, q_{0}\right)$ with an $r$-corner normally oriented by $\xi_{0}$, defines a regular $r$-cubic configuration in $\left(T^{*} M \backslash 0, \eta_{0}\right)$.

Proposition 3.3 Let $V^{0}$ be the hypersurface germ with an $r$-corner in 
$\left(M, q_{0}\right)$ satisfying $\left.\xi_{0}\right|_{T_{q_{0}} V^{0}}=0$ defined as the image of an immersion $\iota$ : $\left(\mathbf{H}^{r} \times \mathbf{R}^{k}, 0\right) \rightarrow\left(M, q_{0}\right)$. Let $\Lambda_{\sigma}$ be the set of rays incident from $V_{\sigma}^{0}:=$ $V^{0} \cap\left\{x_{\sigma}=0\right\}$ at time $t_{0}$ for $\sigma \subset I_{r}$. Then $F:=\tau \circ\left(\iota \times i d_{u}\right)-t_{0} \in \mathrm{m}(r ; k+$ $m)$ is a generating family of the regular $r$-cubic configuration $\left\{\Lambda_{\sigma}\right\}_{\sigma \subset I_{r}}$ in $\left(T^{*} M \backslash 0, \eta_{0}\right)$.

In this case we call $\left\{\Lambda_{\sigma}\right\}_{\sigma \subset I_{r}}$ the regular $r$-cubic configuration associated with $V^{0}$ at time $t_{0}$ and we call the caustic of $\left\{\Lambda_{\sigma}\right\}_{\sigma \subset I_{r}}$ the optical caustic with the r-corner associated with $V^{0}$ at time $t_{0}$.

Proof. By [5, p. 171 Sublemma] we have

$$
\left(\begin{array}{c}
d_{u} d_{x} F \\
d_{u} F
\end{array}\right): T_{u_{0}} M \rightarrow T_{q_{0}}^{*} V^{0} \oplus \mathbf{R}
$$

is an isomorphism. This means that

$$
\operatorname{rank}\left(\begin{array}{c}
\frac{\partial^{2} F}{\partial x \partial u} \\
\frac{\partial^{2} F}{\partial y \partial u}
\end{array}\right)_{0}=r+k .
$$

Hence $F$ is non-degenerate.

Let $\sigma \subset I_{r}$ and $\eta_{u} \in\left(E, \eta_{0}\right)$. Then $\eta_{u} \in \Lambda_{\sigma}$ if and only if $\eta_{u}=$ $\pi_{E} \circ \Phi\left(t, \xi_{q}\right)$ for some $\xi_{q} \in E_{q}$ and $t \in\left(\mathbf{R}, t_{0}\right)$ satisfying $q \in V_{\sigma}^{0}$ and $\left.\xi_{q}\right|_{T_{q} V_{\sigma}^{0}}=0$, if and only if $\eta_{u}=d_{u} \tau(q, u)$ for some $q \in V_{\sigma}^{0}$ and $u \in\left(M, u_{0}\right)$ satisfying $\left.d_{q} \tau(q, u)\right|_{T_{q} V_{\sigma}^{0}}=0$, and this holds if and only if $\eta_{u}=d_{u} F(x, y, u)$ for some $(x, y, u) \in\left(\mathbf{H}^{r} \times \mathbf{R}^{k+m}, 0\right)$ satisfying $x_{\sigma}=0$ and $d_{x_{I_{r}-\sigma}} F(x, y, u)=$ $d_{y} F(x, y, u)=0$. Hence $\left.F\right|_{x_{\sigma}=0}$ is a generating family of $\Lambda_{\sigma}$.

\section{Stability of unfoldings}

In order to investigate stabilities of an optical caustic with an $r$-corner, we require some results of the singularity theory of function germs with respect to reticular $R^{+}$-equivalence. Basic techniques we used in this paper depend heavily on the results in this section, however the all arguments are almost parallel along the ordinary theory of the right-equivalence (cf., [2], $[9])$, so that we omit the detail.

We denote by $J^{l}(r+k, 1)$ the set of $l$-jets at 0 of germs in $\mathrm{m}(r ; k)$ and by $\pi_{l}: \mathrm{m}(r ; k) \rightarrow J^{l}(r+k, 1)$ the natural projection. We denote by $j^{l} f(0)$ 
the $l$-jet of $f \in \mathrm{m}(r ; k)$.

Lemma 4.1 Let $f \in \mathrm{m}(r ; k)$ and $O_{r R}^{l}\left(j^{l} f(0)\right)$ be the submanifold of $J^{l}(r+k, 1)$ consist of the image by $\pi_{l}$ of the orbit of reticular $R$-equivalence of $f$. Put $z=j^{l} f(0)$. Then

$$
\begin{aligned}
T_{z}\left(O_{r R}^{l}(z)\right)=\pi_{l}( & \left\langle x_{1} \frac{\partial f}{\partial x_{1}}, \ldots, x_{r} \frac{\partial f}{\partial x_{r}}\right\rangle_{\mathcal{E}(r ; k)} \\
& \left.+\mathrm{m}(r ; k)\left\langle\frac{\partial f}{\partial y_{1}}, \ldots, \frac{\partial f}{\partial y_{k}}\right\rangle\right) .
\end{aligned}
$$

We say that a function germ $f \in \mathrm{m}(r ; k)$ is reticular $R$-l-determined if all function germ which has same $l$-jet of $f$ is reticular R-equivalent to $f$.

Lemma 4.2 If $f \in \mathrm{m}(r ; k)$ and if

$$
\begin{aligned}
\mathrm{m}(r ; k)^{l+1} \subset \mathrm{m} & (r ; k)\left(\left\langle x_{1} \frac{\partial f}{\partial x_{1}}, \ldots, x_{r} \frac{\partial f}{\partial x_{r}}\right\rangle\right. \\
& \left.+\mathrm{m}(r ; k)\left\langle\frac{\partial f}{\partial y_{1}}, \ldots, \frac{\partial f}{\partial y_{k}}\right\rangle\right)+\mathrm{m}(r ; k)^{l+2},
\end{aligned}
$$

then $f$ is reticular $R$-l-determined. Conversely if $f \in \mathrm{m}(r ; k)$ is reticular $R$-l-determined, then

$$
\mathrm{m}(r ; k)^{l+1} \subset\left\langle x_{1} \frac{\partial f}{\partial x_{1}}, \ldots, x_{r} \frac{\partial f}{\partial x_{r}}\right\rangle_{\mathcal{E}(r ; k)}+\mathrm{m}(r ; k)\left\langle\frac{\partial f}{\partial y_{1}}, \ldots, \frac{\partial f}{\partial y_{k}}\right\rangle .
$$

For each $f(x, y) \in \mathrm{m}(r ; k)^{2}$ we define the corank of $f$ by the corank of the matrix $\left(\frac{\partial^{2} f}{\partial y^{2}}(0)\right)$.

Lemma 4.3 (Splitting lemma) Let $f \in \mathrm{m}(r ; k)^{2}$ and $l$ be the corank of $f$. Then there exist a function germ $f_{0} \in \mathrm{m}(r ; l)^{2}$ and a non-degenerate quadratic form $Q\left(y_{l+1}, \ldots, y_{k}\right)$ such that $\left.f_{0}\right|_{x=0} \in m(0 ; l)^{3}$ and $f$ is reticular $R$-equivalent to $f_{0}\left(x_{1}, \ldots, x_{r}, y_{1}, \ldots, y_{l}\right)+Q\left(y_{l+1}, \ldots, y_{k}\right)$.

Let $F \in \mathrm{m}\left(r ; k+n_{1}\right), G \in \mathrm{m}\left(r ; k+n_{2}\right)$ be unfoldings of $f \in \mathrm{m}(r ; k)$. We say that $F$ is reticular $R^{+}-f$-induced from $G$ if there exist smooth map germs $\phi:\left(\mathbf{H}^{r} \times \mathbf{R}^{k+n_{2}}, 0\right) \rightarrow\left(\mathbf{H}^{r} \times \mathbf{R}^{k}, 0\right), \psi:\left(\mathbf{R}^{n_{2}}, 0\right) \rightarrow\left(\mathbf{R}^{n_{1}}, 0\right)$ and $\alpha \in \mathrm{m}\left(0 ; n_{2}\right)$ satisfying the following conditions:

(1) $\phi\left(\left(\mathbf{H}^{r} \cap\left\{x_{\sigma}=0\right\}\right) \times \mathbf{R}^{k+n_{2}}\right) \subset\left(\mathbf{H}^{r} \cap\left\{x_{\sigma}=0\right\}\right) \times \mathbf{R}^{k}$ for $\sigma \subset I_{r}$.

(2) $G(x, y, v)=F(\phi(x, y, v), \psi(v))+\alpha(v)$ for $x \in \mathbf{H}^{r}, y \in \mathbf{R}^{k}$ and 
$v \in \mathbf{R}^{n_{2}}$.

Definition 4.4 Here we define several notions of stabilities of unfoldings. Let $f \in \mathrm{m}(r ; k)$ and $F \in \mathrm{m}(r ; k+n)$ be an unfolding of $f$.

We define a smooth map germ

$$
j_{1}^{l} F:\left(\mathbf{R}^{r+k+n}, 0\right) \longrightarrow\left(J^{l}(r+k, 1), j^{l} f(0)\right)
$$

as follows: Let $\tilde{F}: U \rightarrow \mathbf{R}$ be a representative of $F$. For each $(x, y, u) \in$ $U$, We define $F_{(x, y, u)} \in \mathrm{m}(r ; k)$ by $F_{(x, y, u)}\left(x^{\prime}, y^{\prime}\right)=F\left(x+x^{\prime}, y+y^{\prime}, u\right)-$ $F(x, y, u)$. Now define $j_{1}^{l} F(x, y, u)$ as the $l$-jet of $F_{(x, y, u)} . \quad j_{1}^{l} F$ depends only on the germ at 0 of $F$. We say that $F$ is reticular $R^{+}-l$-transversal if $\left.j_{1}^{l} F\right|_{x=0}$ is transversal to $O_{r R}^{l}\left(j^{l} f(0)\right)$. It is easy to check that $F$ is reticular $\mathrm{R}^{+}-l$-transversal if and only if

$$
\mathcal{E}(r ; k)=\left\langle x_{1} \frac{\partial f}{\partial x_{1}}, \ldots, x_{r} \frac{\partial f}{\partial x_{r}}, \frac{\partial f}{\partial y_{1}}, \ldots, \frac{\partial f}{\partial y_{k}}\right\rangle_{\mathcal{E}(r ; k)}+V_{F}+\mathrm{m}(r ; k)^{l+1},
$$

where $V_{F}=L_{\mathbf{R}}\left\langle 1,\left.\frac{\partial F}{\partial u_{1}}\right|_{u=0}, \ldots,\left.\frac{\partial F}{\partial u_{n}}\right|_{u=0}\right\rangle$.

We say that $F$ is reticular $R^{+}$-stable if the following condition holds: For any neighborhood $U$ of 0 in $\mathbf{R}^{r+k+n}$ and any representative $\tilde{F} \in C^{\infty}(U, \mathbf{R})$ of $F$, there exists a neighborhood $N_{\tilde{F}}$ of $\tilde{F}$ such that for any element $\tilde{G} \in N_{\tilde{F}}$ the germ $\left.\tilde{G}\right|_{\mathbf{H}^{r} \times \mathbf{R}^{k+n}}$ at $\left(0, y_{0}, u_{0}^{\prime}\right)$ is reticular $\mathrm{R}^{+}$-equivalent to $F$ for some $\left(0, y_{0}, u_{0}^{\prime}\right) \in U$.

We say that $F$ is reticular $R^{+}$-versal if $F$ is reticular $\mathrm{R}^{+}-f$-induced from all unfolding of $f$.

We say that $F$ is reticular $R^{+}$-infinitesimal versal if

$$
\mathcal{E}(r ; k)=\left\langle x_{1} \frac{\partial f}{\partial x_{1}}, \ldots, x_{r} \frac{\partial f}{\partial x_{r}}, \frac{\partial f}{\partial y_{1}}, \ldots, \frac{\partial f}{\partial y_{k}}\right\rangle_{\mathcal{E}(r ; k)}+V_{F}
$$

Theorem 4.5 Let $F \in \mathrm{m}(r ; k+n)$ be an unfolding of $f \in \mathrm{m}(r ; k)$. Then the following are equivalent.

(1) $F$ is reticular $R^{+}$-stable.

(2) $F$ is reticular $R^{+}$-versal.

(3) $F$ is reticular $R^{+}$-infinitesimal versal.

For $f \in \mathbf{m}(r ; k)$, we define the reticular $R$-codimension of $f$ by the 
$\mathbf{R}$-dimension of the vector space

$$
\mathcal{E}(r ; k) /\left\langle x_{1} \frac{\partial f}{\partial x_{1}}, \ldots, x_{r} \frac{\partial f}{\partial x_{r}}, \frac{\partial f}{\partial y_{1}}, \ldots, \frac{\partial f}{\partial y_{k}}\right\rangle_{\mathcal{E}(r ; k)} .
$$

By the above theorem, if 1 and $a_{1}, \ldots, a_{n} \in \mathrm{m}(r ; k)$ is a representative of a basis of the vector space, then $f+a_{1} v_{1}+\cdots a_{n} v_{n} \in \mathrm{m}(r ; k+n)$ is a reticular $\mathrm{R}^{+}$-stable unfolding of $f$.

In [8], we have given the classification of unimodular function germs under the reticular R-equivalence. This classification includes the classification of function germs whose reticular R-codimensions are lower than 8 .

\section{Stability of optical caustics with $r$-corners}

In this section we shall investigate the stability of an optical caustic with an $r$-corner under perturbations of a light source surface with respect to a fixed Hamiltonian function.

Definition 5.1 Let $V^{0}$ be the hypersurface germ in $\left(M, q_{0}\right)$ satisfying $\left.\xi_{0}\right|_{T_{q_{0}} V^{0}}=0$ defined by an immersion $\iota:\left(\mathbf{H}^{r} \times \mathbf{R}^{k}, 0\right) \rightarrow\left(M, q_{0}\right)$. We say that $V^{0}$ produces a stable optical caustic with an $r$-corner at time $t_{0}$ if the following condition holds:

For any open neighborhood $X$ of $q_{0}$ in $M, U$ of $u_{0}$ in $M, W$ of 0 in $\mathbf{R}^{r+k}$, any representative $\tilde{\tau}: X \times U \rightarrow \mathbf{R}$ of $\tau$ and any representative immersion $\tilde{\imath}: W \rightarrow X$ of $\iota$, there exists an open neighborhood $N_{\tilde{\iota}}$ of $\tilde{\iota}$ in the space of immersions from $W$ to $X$ with $C^{\infty}$-topology such that for every $\tilde{\kappa} \in N_{\tilde{\iota}}$ the regular $r$-cubic configuration associated the light source surface defined by $\left.\tilde{\kappa}\right|_{\mathbf{H}^{r} \times \mathbf{R}^{k}}$ at $\left(0, y_{0}\right)$ is lagrangian equivalent to one associated with $V^{0}$ for some $\left(0, y_{0}\right) \in W$.

We remark that, by Theorem 3.2, the condition defined by changing the part 'the regular $r$-cubic $\cdots$ for some $\left(0, y_{0}\right) \in W$ ' in Definition 5.1 to ' $\left.\left(\tilde{\tau} \circ\left(\tilde{\kappa} \times i d_{u}\right)-t_{0}\right)\right|_{\mathbf{H}^{r} \times \mathbf{R}^{k+m}}$ at $\left(0, y_{0}, u_{0}^{\prime}\right)$ is reticular $\mathrm{R}^{+}$-equivalent to $\tau \circ\left(\iota \times i d_{u}\right)-t_{0}$ for some $\left(0, y_{0}, u_{0}^{\prime}\right) \in W \times U^{\prime}$ is equivalent to the original.

Let $V$ be an open set in $\mathbf{R}^{r+k+m}$ with the coordinates $\left(x_{1}, \ldots, x_{r}\right.$, $\left.y_{1}, \ldots, y_{k}, u_{1}, \ldots, u_{m}\right)$. We define the map

$$
j_{1}^{l}: C^{\infty}(V, \mathbf{R}) \rightarrow C^{\infty}\left(V, J^{l}(r+k, 1)\right)
$$

by setting $j_{F}^{l}(x, y, u)$ as the $l$-jet at 0 of the map $\left(x^{\prime}, y^{\prime}\right) \mapsto\left(F\left(x+x^{\prime}, y+\right.\right.$ 
$\left.\left.y^{\prime}, u\right)-F(x, y, u)\right)$ for $F \in C^{\infty}(V, \mathbf{R})$.

Now we give the affirmative answer to the probrem (1).

Theorem 5.2 Let $M$ be an $m(=r+k+1)$-dimensional differentiable manifold, $H: T^{*} M \backslash 0 \rightarrow \mathbf{R}$ a positive and positively homogeneous Hamilton function, $q_{0} \in M, \xi_{0} \in E_{q_{0}}, t_{0} \geq 0$ and $\tau$ the ray length function associated with the regular point $\left(t_{0}, \xi_{0}\right)$ of $\exp _{q_{0}}$. Let $V^{0}$ be the hypersurface germ in $\left(M, q_{0}\right)$ satisfying $\left.\xi_{0}\right|_{T_{q_{0}} V^{0}}=0$ defined by an immersion $\iota:\left(\mathbf{H}^{r} \times \mathbf{R}^{k}, 0\right) \rightarrow$ $\left(M, q_{0}\right)$. Then $V^{0}$ produces a stable optical caustic with an $r$-corner at time $t_{0}$ if and only if $F:=\tau \circ\left(\iota \times i d_{u}\right)-t_{0}$ is a reticular $R^{+}$-versal unfolding of $\left.F\right|_{u=u_{0}}$.

By the above remark, this theorem asserts that the stability of $F$ with respect to perturbations of $\iota$ is sufficient to one of $F$ as an $m$-dimensional unfolding. However generally these stabilities are not equivalent. Since the stability as an unfolding means the stability with respect to both of perturbations of the corresponding light source surface and the Hamiltonian function.

Proof. $\quad(\Leftarrow)$ Let $\tilde{\iota}: W \rightarrow X$ be a representative immersion of $\iota$ and $\tilde{\tau}: X \times U \rightarrow \mathbf{R}$ be a representative of $\tau$. By shrinking $X$ and $U$ if necessary, we may assume that $\left.\tilde{\tau}\right|_{X \times u}$ is submersion for every $u \in U$. We denote $\operatorname{Imm}(W, X)$ the set of immersions from $W$ to $X$ and define the continuous map

$$
\begin{array}{cl}
\Phi: \operatorname{Imm}(W, X) & \longrightarrow C^{\infty}(W \times U, \mathbf{R}) \\
\tilde{\kappa} & \longmapsto \tilde{\tau} \circ\left(\tilde{\kappa} \times i d_{u}\right)-t_{0} .
\end{array}
$$

Set $\tilde{F}=\Phi(\tilde{\imath})$. Since $F$ is a reticular $\mathrm{R}^{+}$-stable unfolding of $f$, there exists a neighborhood $N_{\tilde{F}}$ of $\tilde{F}$ such that, for every function $\tilde{G} \in N_{\tilde{F}}$, the germ $\left.\tilde{G}\right|_{\mathbf{H}^{r} \times \mathbf{R}^{k+m}}$ at $\left(0, y_{0}, u_{0}^{\prime}\right)$ and $F$ are reticular $\mathrm{R}^{+}$-equivalent for some $\left(0, y_{0}, u_{0}^{\prime}\right) \in W \times U$. Then $\Phi^{-1}\left(N_{\tilde{F}}\right)$ is a neighborhood of $\tilde{\iota}$ for which the condition in Definition 5.1 holds.

$(\Rightarrow)$ We suppose Lemma 5.3. Let $\tilde{\iota^{\prime}}: W^{\prime} \rightarrow X$ be a representative immersion of $\iota$ and $\tilde{\tau}: X \times U \rightarrow \mathbf{R}$ be a representative of $\tau$. Choose a relative compact neighborhood $W$ of 0 in $\mathbf{R}^{r+k}$ such that $\bar{W} \subset W^{\prime}$ and choose a neighborhood $N_{\tilde{\iota}}$ of $\tilde{\iota}:=\left.\tilde{\iota^{\prime}}\right|_{W}$ for which the condition in Definition 5.1 holds. We define 


$$
\begin{aligned}
B_{l}=\left\{\tilde{\kappa} \in C^{\infty}\left(W^{\prime}, X\right) \mid\right. & \left.j_{1}^{l}\left(\tilde{\tau} \circ\left(\tilde{\kappa} \times i d_{u}\right)-t_{0}\right)\right|_{x=0} \\
& \text { is transversal to } \left.O_{r R}^{l}\left(j^{l} f(0)\right)\right\}
\end{aligned}
$$

for each $l \in \mathbf{N}$, Then $B_{l}$ is a residual set in $C^{\infty}\left(W^{\prime}, X\right)$ by Lemma 5.3. Since $C^{\infty}\left(W^{\prime}, X\right)$ is a Baire space, $B:=\bigcap_{l \in \mathbf{N}} B_{l}$ is dense.

Set the open set $O=\left\{\tilde{\kappa} \in C^{\infty}\left(W^{\prime}, X\right)|\tilde{\kappa}|_{W}\right.$ is an immersion $\}$. Then the map $O \rightarrow \operatorname{Imm}(W, X)$ given by $\left.\tilde{\kappa} \mapsto \tilde{\kappa}\right|_{W}$ is continuous. Therefore the inverse image $N_{\tilde{\iota}^{\prime}}$ of $N_{\tilde{\iota}}$ by the above map is open neighborhood of $\tilde{\iota}^{\prime}$.

Fix $\tilde{\kappa} \in N_{\tilde{\iota}^{\prime}} \cap B$ sufficiently close to $\tilde{\iota}^{\prime}$ such that $\left(\tilde{\tau} \circ\left(\tilde{\kappa} \times i d_{u}\right)-\right.$ $\left.t_{0}\right)\left.\right|_{\mathbf{H}^{r} \times \mathbf{R}^{r+k}}$ at $\left(0, y_{0}, u_{0}^{\prime}\right)$ and $F$ are reticular $\mathrm{R}^{+}$-equivalent at $\left(0, y_{0}, u_{0}^{\prime}\right) \in$ $W \times U$. Define $G \in \mathrm{m}(r ; k+m)$ by $G(x, y, u):=\tilde{\tau}\left(\tilde{\kappa}\left(x, y+y_{0}\right), u+u_{0}^{\prime}\right)-t_{0}$. Then $G$ is reticular $\mathrm{R}^{+}-l$-transversal unfolding of $g:=\left.G\right|_{u=0}$ for all $l \in \mathbf{N}$. Hence $G$ is a reticular $\mathrm{R}^{+}$-versal unfolding of $g$. Therefore $F$ is also a reticular $\mathrm{R}^{+}$-versal unfolding of $f$.

The following completes the proof the Theorem 5.2.

Lemma 5.3 Let $W, X$ and $U$ be neighborhoods of 0 in $\mathbf{R}^{r+k}, \mathbf{R}^{m}$ and $\mathbf{R}^{n}$ respectively and we denote their coordinates $\left(x_{1}, \ldots, x_{r}, y_{1}, \ldots, y_{k}\right)$, $\left(q_{1}, \ldots, q_{m}\right)$ and $\left(u_{1}, \ldots, u_{n}\right)$ respectively. Let $H: X \times U \rightarrow \mathbf{R}$ be a smooth map such that $\left.H\right|_{X \times u}$ is a submersion for all $u \in U$ and $A$ be a submanifold of $J^{l}(r+k, 1)$. Then the set

$$
B=\left\{f \in C^{\infty}(W, X)\left|j_{1}^{l} H \circ\left(f \times i d_{u}\right)\right|_{x=0} \text { is transversal to } A\right\}
$$

is residual.

Proof. Let $V=W \cap\{x=0\}$. Then the map

$$
\begin{aligned}
\gamma: C^{\infty}(W, X) \rightarrow C^{\infty}\left(V \times U, J^{l}(r+k, 1)\right) \\
\left(\left.f \mapsto j_{1}^{l}\left(H \circ\left(g \times i d_{u}\right)\right)\right|_{x=0}\right)
\end{aligned}
$$

is continuous. If $K \subset A$ is a compact subset, then $C=\left\{F \in C^{\infty}(V \times\right.$ $\left.U, J^{l}(r+k, 1)\right) \mid F$ is transversal to $A$ on $\left.K\right\}$ is open. Therefore $B=\gamma^{-1}(C)$ is open.

Choose relatively compact open covering $\left\{W_{i}\right\}_{i \in \mathbf{N}}$ and $\left\{W_{i}^{\prime}\right\}_{i \in \mathbf{N}}$ of $W$ such that $\overline{W_{i}} \subset W_{i}^{\prime}$ for $i \in \mathbf{N}$. For each $i \in \mathbf{N}$ set

$$
\begin{aligned}
B_{i}=\left\{f \in C^{\infty}(W, X) \mid\right. & \left.j_{1}^{l} H \circ\left(f \times i d_{u}\right)\right|_{x=0} \\
& \text { is transversal to } \left.A \text { on } \bar{W}_{i} \cap\{x=0\}\right\} .
\end{aligned}
$$


Since $B=\bigcap_{i \in \mathbf{N}} B_{i}$ and each $B_{i}$ is open by an analogous proof of the above, it is enough to prove that every $B_{i}$ is dense in order to complete the proof.

The proof is analogous to that of ordinary transversal lemma. Fix $i \in \mathbf{N}$ and $f \in C^{\infty}(W, X)$. Let $P$ be the set of all $n$-tuples of polynomial maps of degree $\leq l$ on $x, y$. Choose a smooth function $\rho: W \rightarrow[0,1]$ such that $\rho=1$ on $\overline{W_{i}}$ and $\rho=0$ on $W-W_{i}^{\prime}$. Put $P^{\prime}=\{\alpha \in P \mid(f+\rho \cdot \alpha)(W) \subset X\}$. Since $P^{\prime}=\left\{\alpha \in P \mid(f+\rho \cdot \alpha)\left(W_{i}^{\prime}\right) \subset X\right\}$ and $\bar{W}_{i}$ is compact, $P^{\prime}$ is a neighborhood of 0 . We define the following maps for $\alpha \in P^{\prime}$ :

$$
\begin{aligned}
& \iota_{\alpha}: V \times U \longrightarrow W^{\prime} \times U \times P^{\prime}((y, u) \mapsto(y, u, \alpha)) \\
& \begin{aligned}
\mu: V \times U \times P^{\prime} \longrightarrow J^{l}(r+k, 1) \\
\left.\quad\left((y, u, \alpha) \mapsto j_{1}^{l}\left(H \circ\left((f+\rho \cdot \alpha) \times i d_{u}\right)\right)(0, y, u)\right)\right) .
\end{aligned}
\end{aligned}
$$

Let $\alpha \in P^{\prime}$. Then $(f+\rho \cdot \alpha) \in B_{i}$ if and only if $\left.j_{1}^{l}\left(H \circ\left((f+\rho \cdot \alpha) \times i d_{u}\right)\right)\right|_{x=0}$ is transversal to $A$ on $\overline{W_{i}} \cap\{x=0\}$, and this holds if and only if $\mu \circ \iota_{\alpha}$ is transversal to $A$ on $\overline{W_{i}} \cap\{x=0\}$, Since $\rho=1$ on $W_{i}$ and $\left.H\right|_{X \times u}$ is a submersion, $\mu$ is submersion and hence this holds if $\iota_{\alpha}$ is transversal to $A^{\prime}:=\mu^{-1}(A)$. Hence $\iota_{\alpha}$ is transversal to $A$ at $(0, y, u, \alpha) \in V \times U \times P^{\prime}$ if and only if $(0, y, u, \alpha) \notin A^{\prime}$ or the projection $\pi: A^{\prime} \rightarrow P^{\prime}$ is regular at $(0, y, u, \alpha)$.

Since the set of critical values of $\pi$ has measure 0 in $P^{\prime}$ by the SardBrown theorem, there exists $\alpha$ arbitrarily near 0 such that $j_{1}^{l}(H \circ((f+\rho$. $\left.\left.\alpha) \times i d_{u}\right)\right)\left.\right|_{x=0}$ is transversal to $A$ on $\bar{W}_{i} \cap\{x=0\}$. This means that there exists $g \in C^{\infty}(W, X)$ arbitrarily close $f$ such that $\left.j_{1}^{l}\left(H \circ\left(g \times i d_{u}\right)\right)\right|_{x=0}$ is transversal to $A$ on $\bar{W}_{i} \cap\{x=0\}$. Hence $B_{i}$ is dense.

\section{Versality of optical caustics with $r$-corners}

In this section we shall investigate our second problem. Recall that $\tau:\left(M \times M,\left(q_{0}, u_{0}\right)\right) \rightarrow\left(\mathbf{R}, t_{0}\right)$ denotes the ray length function. We say that a function germ $f \in \mathrm{m}(r ; k)^{2}$ occur as an organizer of a reticular versal caustic at $\left(t_{0}, \xi_{0}\right)$ if there exists the hypersurface germ $V^{f}$ in $\left(M, q_{0}\right)$ satisfying $\left.\xi_{0}\right|_{T_{q_{0}} V^{0}}=0$ defined by an immersion $\iota_{f}:\left(\mathbf{H}^{r} \times \mathbf{R}^{k}, 0\right) \rightarrow\left(M, q_{0}\right)$ such that $\tau \circ\left(\iota_{f} \times i d_{u}\right)-t_{0}$ is a reticular $\mathrm{R}^{+}$-versal unfolding of $f$.

Lemma 6.1 Let a function germ $f \in \mathrm{m}(r ; k)^{2}$ occur as an organizer of a reticular versal caustic at $\left(t_{0}, \xi_{0}\right)$. If a function germ $g \in \mathrm{m}(r ; k)^{2}$ is reticular $R$-equivalent to $f$, then $g$ also does occur as an organizer of a 
reticular versal caustic at $\left(t_{0}, \xi_{0}\right)$.

Proof. By the hypothesis, there exists a hypersurface germ $V^{f}$ and an immersion $\iota_{f}$ to which above condition holds. Since $f$ is reticular $R$-equivalent to $g$, there exists $\phi \in \mathcal{B}(r, k)$ such that $g=f \circ \phi$. Consider the coordinate change $(x, y) \mapsto \phi^{-1}(x, y)$ on $V^{f}$. Let $V^{g}$ be the hypersurface germ of $\left(M, q_{0}\right)$ parameterized by $\iota_{g}$ :

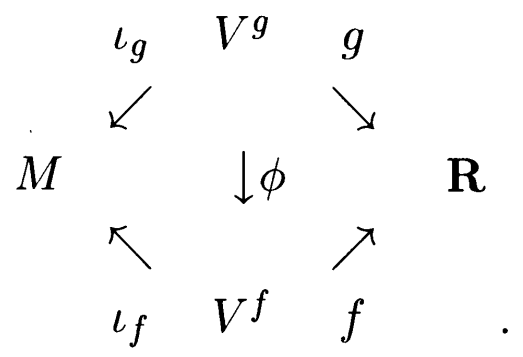

By the above diagram we have

$$
\begin{aligned}
G(x, y, u) & :=\tau\left(\iota_{g}(x, y), u\right)-t_{0}=\tau\left(\iota_{f}(\phi(x, y)), u\right)-t_{0} \\
& =F(\phi(x, y), u) .
\end{aligned}
$$

Since $F$ is reticular $\mathrm{R}^{+}$-versal unfolding of $f, G$ is reticular $\mathrm{R}^{+}$-versal unfolding of $\left.G\right|_{u=0}=f \circ \phi=g$.

Definition 6.2 $[5,3.2]$ Let $u \in M$ and $\eta \in E_{u}$. Then we say that the Hamiltonian function $H$ has rank $s$ at $u$ in direction $\eta$ if the following condition holds: Let $L_{\eta}$ be the line in $T_{u}^{*} M$ spanned by $\eta$. If we introduce affine coordinates $v_{1}, \ldots, v_{m}$ in $T_{u}^{*} M$ such that $T_{\eta} E_{u}$ is given by $v_{m}=1$, the $v_{m}$-axis is $L_{\eta}$, and if we represent $E$ locally at $\eta$ as $v_{m}=1+h\left(v_{1}, \ldots, v_{m-1}\right)$, then the Hessian of $h$ at $\eta$ has rank $s$.

Theorem 6.3 Let $M$ be an $m(=r+k+1)$-dimensional differentiable manifold, $H: T^{*} M \backslash 0 \rightarrow \mathbf{R}$ a positive and positively homogeneous Hamilton function, $q_{0} \in M, \xi_{0} \in E_{q_{0}}$ and $t_{0} \geq 0$. Assume that $\left(t_{0}, \xi_{0}\right)$ is a regular point of $\exp _{q_{0}}$, put $u_{0}=\exp _{q_{0}}\left(t_{0}, \xi_{0}\right)$ and suppose $\eta_{0} \in E_{u_{0}}$ be the image of $\xi_{0}$ under the local flow of $H$ at time $t_{0}$. Then each of following conditions (1), (2) is sufficient for $f \in \mathrm{m}(r ; k)^{2}$ to occur as an organizer of a reticular versal caustic at $\left(t_{0}, \xi_{0}\right)$ :

(1) The reticular $R$-codimension $f \leq m$.

(2) The reticular $R$-codimension $f=m+1$, corank $f \geq 1$ and the rank $s$ of $H$ at $u_{0}$ in direction $\eta_{0} \geq 1$. 
Proof. Choose coordinates $\left(u_{1}, \ldots, u_{m}\right)$ of $M$ at $u_{0}$ such that, with respect to the corresponding fiber coordinates $\left(v_{1}, \ldots, v_{m}\right)$ in $T_{u_{0}}^{*} M, H$ satisfies the conditions in Definition 6.2. By a linear coordinate change of $\left(u_{1}, \ldots, u_{m-1}\right)$, we may assume that $h$ has the form $h\left(v_{1}, \ldots, v_{m}\right)=$ $\sum_{i=1}^{r} \varepsilon_{i} v_{i}^{2}+\sum_{j=1}^{k} \delta_{j} v_{r+j}^{2}+a$, where $\varepsilon_{i}, \delta_{j}=0$ or $\pm 1, a \in \mathrm{m}(r ; k)^{3}$ and in the case $(2) \delta_{1} \neq 0$.

Let $f \in \mathrm{m}(r ; k)^{2}$ satisfy the condition (1) or (2). By Splitting Lemma 4.3 , there exists a function germ $f_{0} \in \mathrm{m}(r ; l)^{2}$ such that $f$ is reticular Requivalent to $f_{0}\left(x_{1}, \ldots, x_{r}, y_{1}, \ldots, y_{l}\right) \pm y_{l+1}^{2} \pm \cdots \pm y_{k}^{2}$ and $\left.f_{0}\right|_{x=0} \in \mathrm{m}(0 ; l)^{3}$. We may assume that $f=f_{0} \pm y_{l+1}^{2} \pm \cdots \pm y_{k}^{2}$ by Lemma 6.1. Then we have $\mathcal{E}(r ; k) /\left\langle x \frac{\partial f}{\partial x}, \frac{\partial f}{\partial y}\right\rangle=\mathcal{E}(r ; l) /\left\langle x \frac{\partial f_{0}}{\partial x}, \frac{\partial f_{0}}{\partial y}\right\rangle$.

First, we prove that $x_{1}, \ldots, x_{r}, y_{1}, \ldots, y_{l}$ are linearly independent in $\mathcal{E}(r ; l) /\left\langle x \frac{\partial f_{0}}{\partial x}, \frac{\partial f_{0}}{\partial y}\right\rangle$ over R. Let $\alpha_{1} x_{1}+\cdots+\alpha_{r} x_{r}+\beta_{1} y_{1}+\cdots+\beta_{l} y_{l}=$ $0 \in \mathcal{E}(r ; l) /\left\langle x \frac{\partial f_{0}}{\partial x}, \frac{\partial f_{0}}{\partial y}\right\rangle$ for $\alpha_{1}, \ldots, \alpha_{r}, \beta_{1}, \ldots, \beta_{l} \in \mathbf{R}$. Since $\langle\beta, y\rangle=0$ in $\mathcal{E}(0 ; l) /\left\langle\left.\frac{\partial f_{0}}{\partial y}\right|_{x=0}\right\rangle$ and $\left.f_{0}\right|_{x=0} \in m^{3}(0 ; l)$, we have $\beta=0$. Suppose that $\alpha_{1} \neq 0$. Then there exist $\gamma_{0}, \ldots, \gamma_{l} \in \mathrm{m}(1 ; l)$ such that

$$
\begin{aligned}
& x_{1}+\gamma_{0}\left(x_{1}, y\right) x_{1} \frac{\partial f_{0}}{\partial x_{1}}\left(x_{1}, 0, y\right)+\gamma_{1}\left(x_{1}, y\right) \frac{\partial f_{0}}{\partial y_{1}}\left(x_{1}, 0, y\right) \\
& +\gamma_{l}\left(x_{1}, y\right) \frac{\partial f_{0}}{\partial y_{l}}\left(x_{1}, 0, y\right)=0 .
\end{aligned}
$$

Therefore we have $\gamma_{i}(0) \neq 0$ for some $i \geq 1$, for $x_{1} \frac{\partial f_{0}}{\partial x_{1}}\left(x_{1}, 0, y\right) \in \mathrm{m}(1 ; l)^{2}$. We may assume that $i=1$. Then this means that $\left.\frac{\partial f_{0}}{\partial y_{1}}\right|_{x=0} \in\left\langle\left.\frac{\partial f_{0}}{\partial y_{2}}\right|_{x=0}, \ldots\right.$, $\left.\left.\frac{\partial f_{0}}{\partial y_{l}}\right|_{x=0}\right\rangle$ and contradicts that $\operatorname{dim}_{\mathbf{R}} \mathrm{m}(0 ; l) /\left\langle\left.\frac{\partial f_{0}}{\partial y}\right|_{x=0}\right\rangle<\infty$.

Consider the case (2). The vector space

$$
\mathrm{m}(r ; l) /\left(\left\langle x \frac{\partial f_{0}}{\partial x}, \frac{\partial f_{0}}{\partial y}\right\rangle+L_{\mathbf{R}}\langle x, y\rangle+\mathrm{m}(r ; l)^{3}\right)
$$

must have a positive dimension because if not we have reticular R-codimension $f \leq m$. Therefore we may assume by Lemma 6.1 and Lemma 6.4 below that

$$
\begin{aligned}
b_{k+1}:=\sum_{i=1}^{r} \varepsilon_{i} x_{i}^{2}+\sum_{j=1}^{l} \delta_{j} y_{j}^{2} \neq 0 & \text { in } \\
& \mathrm{m}(r ; l) /\left(\left\langle x \frac{\partial f_{0}}{\partial x}, \frac{\partial f_{0}}{\partial y}\right\rangle+L_{\mathbf{R}}\langle x, y\rangle\right) .
\end{aligned}
$$


Now choose $b_{l+1}, \ldots, b_{t} \in \mathrm{m}(r ; l)^{2}$ and $b_{t+1}, \ldots, b_{k} \in \mathrm{m}(r ; l)^{3}$ such that $x_{1}, \ldots, x_{r}, y_{1}, \ldots, y_{l}, b_{l+1}, \ldots, b_{t}, b_{k+1}$ is a basis of $\mathrm{m}(r ; l) /\left(\left\langle x \frac{\partial f_{0}}{\partial x}, \frac{\partial f_{0}}{\partial y}\right\rangle+\right.$ $\left.\mathrm{m}(r ; l)^{3}\right)$ and $x_{1}, \ldots, x_{r}, y_{1}, \ldots, y_{l}, b_{l+1}, \ldots, b_{k+1}$ is a basis of $\mathrm{m}(r ; l) /$ $\left\langle x \frac{\partial f_{0}}{\partial x}, \frac{\partial f_{0}}{\partial y}\right\rangle$.

In the case $(1)$, choose $b_{l+1}, \ldots, b_{k}$ such that $x_{1}, \ldots, x_{r}, y_{1}, \ldots, y_{l}$, $b_{l+1}, \ldots, b_{k}$ generate $\mathrm{m}(r ; l) /\left\langle x \frac{\partial f_{0}}{\partial x}, \frac{\partial f_{0}}{\partial y}\right\rangle$ over $\mathbf{R}$.

Now define $\phi \in \mathcal{B}(r ; k)$ by

$$
\begin{aligned}
& \phi\left(x_{1}, \ldots, x_{r}, y_{1}, \ldots, y_{k}\right) \\
& \quad=\left(x_{1}, \ldots, x_{r}, y_{1}, \ldots, y_{l}, y_{l+1}+b_{l+1}, \ldots, y_{k}+b_{k}\right) .
\end{aligned}
$$

Since $\exp _{u_{0}}^{-}$is invertible, the map $\iota_{f}:\left(\mathbf{H}^{r} \times \mathbf{R}^{k}, 0\right) \rightarrow\left(M, q_{0}\right)$ given by $\iota_{f}(x, y)=\exp _{u_{0}}^{-}\left(f(x, y)+t_{0},(\phi(x, y), 1+h \circ \phi(x, y))\right)$ defines a hypersurface germ $V_{f}$ in $\left(M, q_{0}\right)$. Then we have

$$
\begin{aligned}
& F(x, y, 0):=\tau\left(\iota_{f}(x, y), u_{0}\right)-t_{0}=\left(f(x, y)+t_{0}\right)-t_{0}=f(x, y), \\
& \left.\xi\left(q_{0}, u_{0}\right)\right|_{T_{q_{0}} V^{f}}=-d_{(x, y)}\left(\tau \circ \iota_{f}\right)\left((0,0), u_{0}\right)=-d_{(x, y)} f(0,0)=0, \\
& d_{u} F(x, y, 0) \\
& \quad=d_{u} \tau\left(\iota_{f}(x, y), u_{0}\right)=\eta\left(\iota_{f}(x, y), u_{0}\right)=(\phi(x, y), 1+h \circ \phi(x, y)) \\
& \quad=\left(x_{1}, \ldots, x_{r}, y_{1}, \ldots, y_{l}, y_{l+1}+b_{l+1}, \ldots, y_{k}+b_{k}, b_{k+1}+a+1\right),
\end{aligned}
$$

where $a \in \mathrm{m}(r ; k)^{3}$.

In the case (1), we have

$$
\begin{aligned}
\mathcal{E}(r ; k) & /\left\langle x \frac{\partial f}{\partial x}, \frac{\partial f}{\partial y}\right\rangle \\
& =L_{\mathbf{R}}\left\langle 1, x_{1}, \ldots, x_{r}, y_{1}, \ldots, y_{l}, b_{l+1}, \ldots, b_{k}\right\rangle \\
& =L_{\mathbf{R}}\left\langle 1, x_{1}, \ldots, x_{r}, y_{1}, \ldots, y_{l}, y_{l+1}+b_{l+1}, \ldots, y_{k}+b_{k}\right\rangle .
\end{aligned}
$$

Hence the proof of the case (1) is completed.

In the case (2). since $1, x_{1}, \ldots, x_{r}, y_{1}, \ldots, y_{l}, b_{l+1}, \ldots, b_{k}, b_{k+1}$ is a basis of $\mathcal{E}(r ; k) /\left\langle x \frac{\partial f}{\partial x}, \frac{\partial f}{\partial y}\right\rangle$, there exist $\alpha_{0}, \alpha_{1}, \ldots, \alpha_{r}, \beta_{1}, \ldots, \beta_{k+1} \in \mathbf{R}$ such that

$$
\begin{aligned}
a \equiv \alpha_{0}+\alpha_{1} x_{1}+\cdots+\alpha_{r} x_{r}+\beta_{1} y_{1}+\cdots+\beta_{l} y_{l}+\beta_{l+1} b_{l+1} \\
+\cdots+\beta_{k+1} b_{k+1} \bmod \mathcal{E}(r ; k) /\left\langle x \frac{\partial f}{\partial x}, \frac{\partial f}{\partial y}\right\rangle .
\end{aligned}
$$


Hence

$$
\begin{array}{r}
0 \equiv \alpha_{0}+\alpha_{1} x_{1}+\cdots+\alpha_{r} x_{r}+\beta_{1} y_{1}+\cdots+\beta_{l} y_{l}+\beta_{l+1} b_{l+1} \\
+\cdots+\beta_{t} b_{t}+\beta_{k+1} b_{k+1} \\
\bmod \mathcal{E}(r ; k) /\left(\left\langle x \frac{\partial f}{\partial x}, \frac{\partial f}{\partial y}\right\rangle+\mathrm{m}(r ; k)^{3}\right)
\end{array}
$$

Since $x_{1}, \ldots, x_{r}, y_{1}, \ldots, y_{l}, b_{l+1}, \ldots, b_{t}, b_{k+1}$ is a basis of $\mathcal{E}(r ; k) /\left(\left\langle x \frac{\partial f}{\partial x}, \frac{\partial f}{\partial y}\right\rangle+\right.$ $\left.\mathrm{m}(r ; k)^{3}\right)$,

$$
\alpha_{0}=\alpha_{1}=\cdots=\alpha_{r}=\beta_{1}=\cdots=\beta_{t}=\beta_{k+1}=0 .
$$

Hence $a \in L_{\mathbf{R}}\left\langle b_{t+1}, \ldots, b_{k}\right\rangle$ in $\mathcal{E}(r ; k) /\left\langle x \frac{\partial f}{\partial x}, \frac{\partial f}{\partial y}\right\rangle$. This means that

$$
\begin{aligned}
& \mathcal{E}(r ; k) /\left\langle x \frac{\partial f}{\partial x}, \frac{\partial f}{\partial y}\right\rangle \\
& \quad=L_{\mathbf{R}}\left\langle 1, x_{1}, \ldots, x_{r}, y_{1}, \ldots, y_{l}, b_{l+1}, \ldots, b_{k}, b_{k+1}+a\right\rangle .
\end{aligned}
$$

Therefore

$$
\begin{aligned}
\mathcal{E}(r ; k) /\left\langle x \frac{\partial f}{\partial x}, \frac{\partial f}{\partial y}\right\rangle & \\
=L_{\mathbf{R}}\left\langle 1, x_{1}, \ldots, x_{r},\right. & y_{1}, \ldots, y_{l}, y_{l+1} \\
& \left.+b_{l+1}, \ldots, y_{k}+b_{k}, b_{k+1}+a+1\right\rangle .
\end{aligned}
$$

Hence the proof for the case (2) is completed, supposing Lemma 6.4.

It remains to show:

Lemma 6.4 Let $A=\operatorname{diag}\left(\varepsilon_{1}, \ldots, \varepsilon_{r}, \delta_{1}, \ldots, \delta_{l}\right) \in M(r+l, r+l ; \mathbf{R})$, $\varepsilon_{1}, \ldots, \varepsilon_{r}, \delta_{1}, \ldots, \delta_{l}$ are 0 or \pm 1 and $\delta_{1} \neq 0$. Then the set $\mathcal{F}$ of matrices linearly generated by $D \phi(0)^{t} A D \phi(0)$ for all $\phi \in \mathcal{B}(r ; l)$ is equal to that of symmetric matrices in $M(r+l, r+l ; \mathbf{R})$.

Proof. We denote $\varepsilon=\operatorname{diag}\left(\varepsilon_{1}, \ldots, \varepsilon_{r}\right)$ and $\delta=\operatorname{diag}\left(\delta_{1}, \ldots, \delta_{l}\right)$. At first we remark that

$$
\{B \delta C \in M(s, t ; \mathbf{R}) \mid B \in M(s, l ; \mathbf{R}), C \in M(l, t ; \mathbf{R})\}=M(s, t ; \mathbf{R})
$$

for any integer $s$ and $t$. Let $\phi \in \mathcal{B}(r ; l)$ be given. We denote $\phi(x, y)=$ $\left(x_{1} a_{1}(x, y), \ldots, x_{r} a_{r}(x, y), b_{1}(x, y), \ldots, b_{l}(x, y)\right)$. Then we have by immedi- 
ately calculation that

$$
\begin{array}{ll}
D \phi(0)^{t} A D \phi(0)= & \\
& \left(\begin{array}{cc}
\operatorname{diag}\left(a_{1}^{2}(0) \varepsilon_{1}, \ldots, a_{r}^{2}(0) \varepsilon_{r}\right)+2\left(\frac{\partial b}{\partial x}(0)\right)^{t} \delta\left(\frac{\partial b}{\partial x}(0)\right) & 2\left(\frac{\partial b}{\partial x}(0)\right)^{t} \delta\left(\frac{\partial b}{\partial y}(0)\right) \\
2\left(\frac{\partial b}{\partial y}(0)\right)^{t} \delta\left(\frac{\partial b}{\partial x}(0)\right) & 2\left(\frac{\partial b}{\partial y}(0)\right)^{t} \delta\left(\frac{\partial b}{\partial y}(0)\right)
\end{array}\right) .
\end{array}
$$

By considering the case $\frac{\partial b}{\partial x}(0)=0$ we have

$$
\begin{aligned}
& \left\{\left(\begin{array}{rr}
\operatorname{diag}\left(a_{1}^{2}(0) \varepsilon_{1}, \ldots, a_{r}^{2}(0) \varepsilon_{r}\right) & 0 \\
0 & B
\end{array}\right)\right. \\
& \quad \in M(r+l, r+l ; \mathbf{R}) \mid B \in M(l, l ; \mathbf{R})\} \subset \mathcal{F} .
\end{aligned}
$$

This means that

$$
\left\{\left(\begin{array}{cc}
0 & 0 \\
0 & B
\end{array}\right) \in M(r+l, r+l ; \mathbf{R}) \mid B \in M(l, l ; \mathbf{R})\right\} \subset \mathcal{F} .
$$

Let $\phi, \phi^{\prime} \in \mathcal{B}(r ; l)$ satisfy the conditions that $a(0)=a^{\prime}(0), \frac{\partial b}{\partial x}(0)=\frac{\partial b^{\prime}}{\partial x}(0)$, where $\phi(x, y)=\left(x_{1} a_{1}(x, y), \ldots, x_{r} a_{r}(x, y), b_{1}(x, y), \ldots, b_{l}(x, y)\right)$ and $\phi^{\prime}(x, y)=\left(x_{1} a_{1}^{\prime}(x, y), \ldots, x_{r} a_{r}^{\prime}(x, y), b_{1}^{\prime}(x, y), \ldots, b_{l}^{\prime}(x, y)\right)$. Then

$$
\begin{aligned}
& D \phi(0)^{t} A D \phi(0)-D \phi^{\prime}(0)^{t} A D \phi^{\prime}(0) \\
& \quad=\left(\begin{array}{cc}
0 & 2\left(\frac{\partial b}{\partial x}(0)\right)^{t} \delta\left(\frac{\partial b}{\partial y}(0)-\frac{\partial b^{\prime}}{\partial y}(0)\right) \\
2\left(\frac{\partial b}{\partial y}(0)-\frac{\partial b^{\prime}}{\partial y}(0)\right)^{t} \delta\left(\frac{\partial b}{\partial x}(0)\right) & *
\end{array}\right) .
\end{aligned}
$$

Therefore

$$
\left\{\left(\begin{array}{cc}
0 & B \\
B^{t} & 0
\end{array}\right) \in M(r+l, r+l ; \mathbf{R}) \mid B \in M(r, l ; \mathbf{R})\right\} \subset \mathcal{F} .
$$

Similarly we have

$$
\left\{\left(\begin{array}{cc}
B & 0 \\
0 & 0
\end{array}\right) \in M(r+l, r+l ; \mathbf{R}) \mid B \in M(r, r ; \mathbf{R}), B^{t}=B\right\} \subset \mathcal{F} .
$$

Acknowledgments The author would like to thank Professor S. Izumiya for useful remarks and useful discussions. 


\section{References}

[1] Arnold V.I., Gusein-Zade S.M. and Varchenko A.N., Singularities of differential maps. Vol.I, Birkhäuser, Basel, 1986.

[2] Bröcker Th. and Lander L., Differentiable germs and catastrophes. LMS-Lecture note series 17, CUP 1975.

[3] Duc N.H., Dai N.T. and Pham F., Singularités non-dégénérées des systèmes de Gauss-Manin réticulés. Memoire de la S. M. F.,Nouvelle serie n.6, 1981.

[4] Duc N.H., Involutive singularities. Kodai Math. J. 17 (1994), 627-635.

[5] Jänich K., Caustics and catastrophes. Math. Ann. 209 (1974), 161-180.

[6] Matov V.I., Unimodal germs of functions on a manifold with a boundary. Functional. Anal. i Prilozhen 14 (1980) no.1, 69-70.

[7] Scherbak I.G., Boundary fronts and caustics and their metamorphosis. LMSLecture note series 201, Singularities, 363-373.

[ 8 ] Tsukada T., Reticular Lagrangian Singularities. The Asian Journal of Mathematics 1 (1997), 572-622.

[9] Wassermann G., Stability of unfolding. Lecture note in mathematics 393, 1974.

[10] Wassermann G., Stability of caustics. Math. Ann. 216 (1975), 43-50.

Department of Mathematics

Faculty of Science, Hokkaido University

Sapporo 060-0810, Japan

E-mail: ttsukada@math.sci.hokudai.ac.jp 